\title{
Technical innovation: Intragastric Single Port Sleeve Gastrectomy (IGSG). A feasibility survival study on porcine model $^{1}$
}

\author{
Oscar Estupinam', André Lacerda de Abreu Oliveira", Fernanda Antunes'"', Manoel Galvão'v , Henrique \\ Phillips ${ }^{\mathrm{v}}$, Jussara Peters Scheffer ${ }^{\mathrm{VI}}$, Marcelo Rios ${ }^{\mathrm{VII}}$, Ricardo Zorron ${ }^{\mathrm{VIII}}$
}

'MSc in Sciences, Animal Experimentation Division, Laboratory of Animal Health, Center for Agricultural Sciences and Technologies, Universidade Estadual do Norte Fluminense Darcy Ribeiro (UENF), Campos dos Goytacazes-RJ, Brazil. Conception and design of the study, technical procedures, acquisition and analysis of data, manuscript writing.

"PhD in Sciences, Associate Professor, Animal Experimentation Division, Laboratory of Animal Health, Center for Agricultural Sciences and Technologies, UENF Darcy Ribeiro, Campos dos Goytacazes-RJ, Brazil. Critical revision, final approval.

IIIPhD in Sciences, Associate Professor, Animal Experimentation Division, Laboratory of Animal Health, Center for Agricultural Sciences and Technologies, UENF Darcy Ribeiro, Campos dos Goytacazes-RJ, Brazil. Technical procedures.

IVPhD in Sciences, Gastro Obeso Center, Obesity Surgery, Sao Paulo-SP, Brazil. Critical revision.

${ }^{\vee}$ PhD in Sciences, Universidade Federal do Estado do Rio de Janeiro (UNIRIO), Brazil. Technical procedures, manuscript writing.

VIFellow PhD degree, Postgraduate Program in Animal Science, Animal Experimentation Division, Laboratory of Animal Health, Center for Agricultural Sciences and Technologies, UENF Darcy Ribeiro, Campos dos Goytacazes-RJ, Brazil. Conception and design of the estudy, technical procedures, critical revision.

VIIPhD in Sciences, Animal Experimentation Division, Laboratory of Animal Health, Center for Agricultural Sciences and Technologies, UENF Darcy Ribeiro, Campos dos Goytacazes-RJ, Brazil. Technical procedures, critical revision.

VIIIPhD in Sciences, Animal Experimentation Division, Laboratory of Animal Health; Center for Agricultural Sciences and Technologies, UENF Darcy Ribeiro, Campos dos Goytacazes-RJ, Brazil. Technical procedures, critical revision, final approval.

\begin{abstract}
Purpose: To perform technically the laparoscopic sleeve gastrectomy (LSG) using a unique Intragastric Single Port (IGSG) in animal swine model, evidencing an effective and safe procedure, optimizing the conventional technique.

Methods: IGSG was performed in 4 minipigs, using a percutaneous intragastric single port located in the pre-pyloric region. The gastric stapling of the greater curvature started from the pre-pyloric region towards the angle of His by Endo $\mathrm{GIA}^{\mathrm{TM}}$ system and the specimen was removed through the single port. In the postoperative day 30 , the animals were sacrificed and submitted to autopsy.

Results: All procedures were performed without conversion, and all survived 30 days. The mean operative time was $42 \mathrm{~min}$. During the perioperative period no complications were observed during invagination and stapling. No postoperative complications occurred. Postmortem examination showed no leaks or infectious complications.

Conclusion: Intragastric Single Port is a feasible procedure that may be a suitable alternative technique of sleeve gastrectomy for the treatment of morbid obesity.

Key words: Bariatric Surgery. Obesity, Morbid. Laparoscopy.
\end{abstract}




\section{- Introduction}

Since the introduction of laparoscopic Sleeve Gastrectomy (LSG) as the restrictive component of the duodenal switch, different approaches have been described to make it simpler and minimize surgical trauma and complications. Regan et al proposed LSG as a first step procedure for superobese patients ${ }^{1}$, and the concept evolved to LSG as an effective alone standing procedure ${ }^{2,3}$.

Although the acceptance and indication for LSG is growing fast, the procedure still has a complex learning curve and is responsible for significant postoperative morbidity and mortality. Leakage after LSG occurs in about 0.5 to $5.3 \% \%^{4,5}$. Bleeding and haematoma of the staple line are a risk for morbidity and reoperations, thus oversewing and buttressing were also proposed to reduce this occurrence ${ }^{6}$.

To reduce the invasivity and complications of surgery in the morbidly obese patient set, many endoluminal procedures currently aim to minimize the surgical complications and the complexity and cost involved with the therapy, but the clinical use of these therapies is spreading slowly due to the need of flexible endoscopy practice ${ }^{7}$. Single Port surgery is emerging as an attractive simplified technique for laparoscopic surgery for many indications, currently increasingly employed in colorectal, gastric and general surgery, with low complication and conversion rates. The reduction of the number of ports potentially may decrease the occurrence of wound complications, and may evolve to reduce operative time and costs. The use of Single Port to perform sleeve gastrectomy was sporadically described, but didn't gained popularity among the medical community, possibly because of the lack of visualization and retraction ${ }^{8,9}$. Intragastric Single Port surgery has been reported as one of the minimally invasive approaches to access the gastric lumen and being suitable for resecting gastric tumors or extracting larger foreign bodies from the stomach ${ }^{10}$, but not for sleeve gastrectomy.

This study presents a new technique for performing sleeve gastrectomy (IGSGIntragastric Single Port Sleeve Gastrectomy) using previous percutaneous exteriorization of the gastric wall and its feasibility in a survival experimental animal set. The objective of this study is to test the potential of IGSG as an alternative therapy for morbid obesity.

\section{Methods}

The study was approved by the Institutional Animal Care and Use Committee, Universidade Estadual do Norte Fluminense Darcy Ribeiro. All applicable institutional and national guidelines for the care and use of animals were followed.

Four mini pigs weighing 35 to $40 \mathrm{~kg}$ were presented for this study. The animals were fasted except for water intake for 24 hours before operation. After an intramuscularly injected preanesthetic cocktail of hydrochloride acepromazine $(0.1 \mathrm{mg} / \mathrm{kg})$ and ketamine $(1 \mathrm{mg} /$ $\mathrm{kg}$ ), intravenous (IV) access was established with Ringer's solution via a large ear vein, and anesthetic induction was achieved with the IV mixed Propofol (10\%) and sodium thiopental $(2.5 \%)$ in a $1: 1$ proportion, diluted at the same seringe, administered on a dose-effect. They were placed in supine position and secured with straps. After endotracheal intubation, ventilation was achieved with a tidal volume of $15-20 \mathrm{ml} / \mathrm{kg}$ at a rate of $12-18$ breaths/min. Inspired fraction of inspired oxygen $\left(\mathrm{FIO}_{2}\right)$ was monitored for optimal oxygenation. Anesthesia was maintained with isofluorane (1.5\%). Pulse oximeter, end-tidal $\mathrm{CO}_{2}$, blood pressure, and three-lead electrocardiogram were monitored throughout the procedure. 


\section{Surgical technique}

The animals were kept in a supine position, the operator were standing at the right and the assistant on the left side of the animal. The video monitor was placed in a central position, above the head of the animal, in the surgeon's and assistant line of vision. A transesophageal endoscope was inserted for evaluation of gastric cleaness and for previous insufflation. The principle of the technique is the direct insertion of the single port device inside the gastric lumen after exteriorization of the gastric wall, and performing a sleeve gastrectomy from inside (Figure 1).

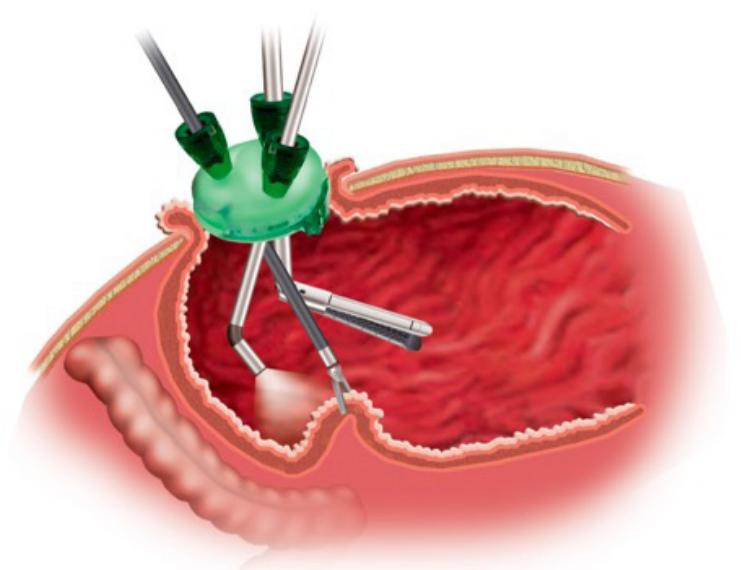

Figure 1 - Schematic view of Intragastric Single Port Sleeve Gastrectomy (IGSG).

The procedure commenced with open entry into the abdomen through a $2-\mathrm{cm}$ supra-umbilical incision on the left side of the abdomen to access the peritoneum (Figure 2A). The stomach is transorally insuflated with air using the gastric tube. After gastric location under direct view without using a camera, the antrum was grasped with a Babcock grasper and tractioned outside the cavity (Figure 2B). The gastric wall was then sutured to the skin without tension with several surrounding Vicryl 2-0 stitches (Figure $2 \mathrm{C}$ ). A 2-cm incision in the pre-pyloric area of the exteriorzed stomach was made using an electrocautery in a coagulation mode. The Single Port device (Olympus TriPort, Olympus OSTE, Germany) was implemented intragastrically and adjusted (Figure 2D).

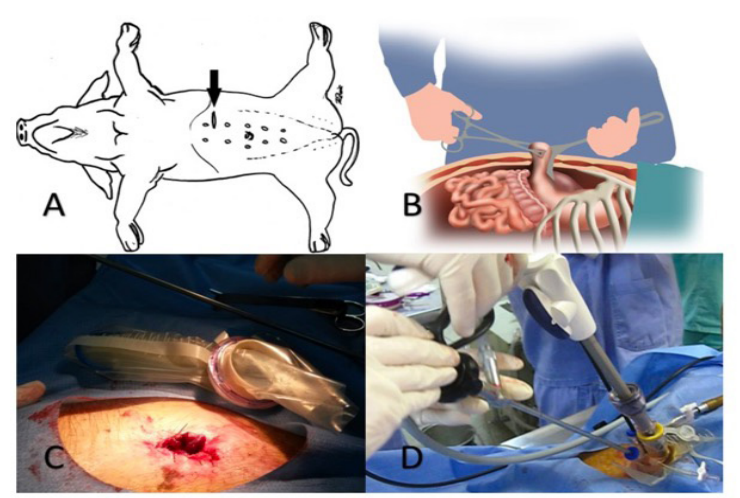

Figure 2 - A. Position of the animal in supine fixation. Arrow demonstrates the site of the only incision in the upper abdomen. B. Schematic view of prior exteriorization of the gastric wall under direct view. C. Operative external view of fixation of the gastric wall to the skin and opening of gastric lumen. D. Operative external view: Insertion of the instruments through Single Port device.

The platform contains one $12 \mathrm{~mm}$ and two $5 \mathrm{~mm}$ ports. Carbon dioxide was inflated into the stomach through the insuflation tube on the access port. Intragastric pressure was maintained at $8 \mathrm{~mm} \mathrm{Hg}$ by a laparoscopic insufflator. A 5-mm $30^{\circ}$ laparoscope, one grasping forceps and the linear Endo $\mathrm{GIA}^{\mathrm{TM}}$ $30 \mathrm{~mm}$ Articulating Medium/Thick Reload with Tri-Staple ${ }^{\mathrm{TM}}$ Technology (Covidien, New Haven) were introduced into the stomach through the working channels on the port. The proximal greater curvature was internally grasped and tractioned to the right, allowing for a maximal amount of gastric tissue to be included in the stapler. The gastric stapling of the greater curvature started from the antral region, progressing cephalad towards the angle of His by successive firing the linear Endo $\mathrm{GIA}^{\mathrm{TM}}$ (Figure 3). 

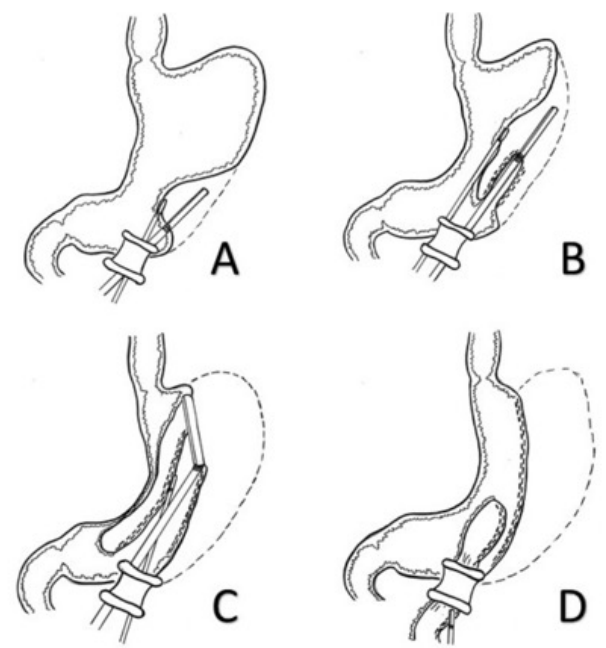

Figure 3 - A. Schematic illustration of internal gastric stapling through percutaneous single port device installed in the pre-pyloric area. Grasping of the gastric antrum and first positioning of the linear stapler. B. Firing the first linear stapler to the fundic area. C. Completion of the stapling of the gastric fundus shortly below the cardia. D. Extraction of the gastric sleeve through the single port device.

The gastric corpus and fundus were tractioned using laparoscopic forceps and the Endo $\mathrm{GIA}^{\mathrm{TM}}$ system was fired (Figure 4A). After the entire staple line was completed, the endoscope was used to evaluate the lumen for hemostasis and patency (Figure 4B). After certification of absence of bleeding, gastric specimen was extracted by the transgastric port (Figure 4C).

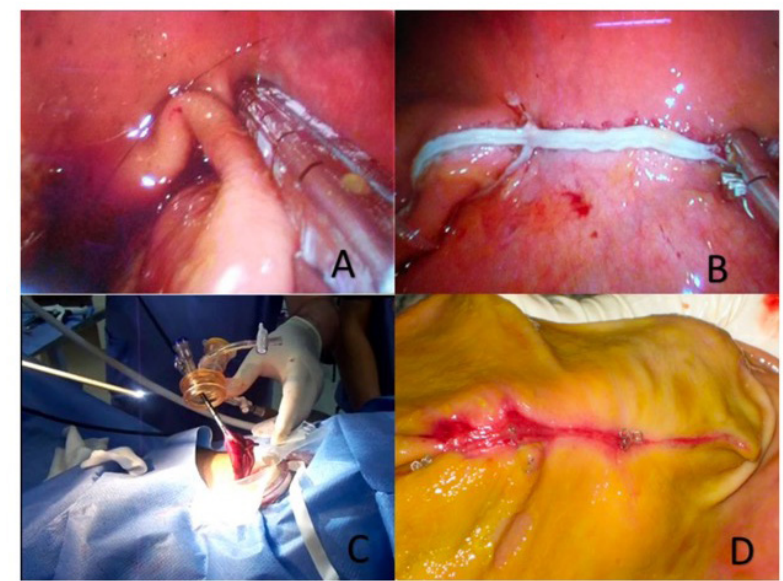

Figure 4 - A. Stapling of the greater curvature of the stomach. B. Final aspect of the stapler line after intraoperative firing. C. Removal of the specimen through the single port. D. Region of gastric stapling after 30 days of surgical procedure.
Essentially, the procedure is performed with a formal sleeve resection of the fundus and greater curvature of the stomach, using linear stapling, but from inside the gastric lumen. There are no vessel ligation from outside, just the full-thickness internal stapling. The final specimen is a tubular sleeve that has to be extracted through the intragastric placed single port device. The gastric closure was performed by the initial incision with 3-0 vicryl suture in a two-layer inverting seromuscular pattern and the aponeurosis and skin were closed with absorbable running sutures as usual. Finally, laparoscopic and endoscopic checking on bleeding and methylene blue test were assessed and the endoscope was removed. The tubular specimens were satisfactory and represented approximately two thirds of the gastric volume in all cases. Measurement of remnant gastric volume was not a primary goal of the study and was not performed, as well as weight measures of the specimens. In this pilot study, the goal was to evaluate the feasibility of an intragastric sleeve resection.

In the post-operative period the animals were treated with non-steroidal anti-inflammatory drug (NSAID), at a dose of $0.1 \mathrm{mg}$ meloxicam/ $\mathrm{kg}$ every 24 hours for 5 days intramuscularly; systemic antibiotic trimethoprim / sulfametaxol at a dose of $48 \mathrm{mg} /$ kg every 24 hours for 10 days by intramuscular route and local antibiotic, silver sulfadiazine spray at the site of the surgical wound. After the complete return of consciousness and for the next 30 days were fed in the usual way to assess signs of anorexia and abdominal pain. After 30 days period, the pigs were sacrificed to perform the macroscopic and microscopic examinations by necropsy and histopathology, and bacterial samples were taken from the peritoneal cavity. Weight loss and remnant gastric volume were not an objective of the study and were not evaluated in this short postoperative survival period. 


\section{Results}

All the procedures were performed without conversion or intraoperative complications. Operative time was a mean of 42 minutes, and the access for the gastric lumen was not time-consuming (Table 1). During the induction and maintenance of anesthesia the animals showed no changes of the evaluated parameters (ECG, capnography, pulse oximetry, temperature, perfusion index, heart rate and respiratory rate.). During the perioperative period, expected hazards as visualization, insuflation or orientation problems, instrument collision, difficulty in gastric traction or bleeding during invagination and stapling were not observed. During the postoperative period the animals showed no abnormalities such as fever, vomiting, anorexia, or other signs that indicate complications. Although changes among which may be cited, adhesions, stenosis, abscesses, gastric fistulas, bleeding may occur in this type of surgical procedure, they were not detected during necropsy at 30 days (Figure 4D) as well as pathological changes in the gastric scar fragments collected for microscopic evaluation.

Table 1 - Intraoperative and postoperative results for IGSG in a porcine survival study.

\begin{tabular}{|c|c|c|}
\hline Results summary & Average & $\begin{array}{l}\text { Standard } \\
\text { deviation }\end{array}$ \\
\hline Access time (minutes) & 7.75 & 2.06 \\
\hline Stapling time (minutes) & 20.75 & 10.90 \\
\hline Closing time (minutes) & 11 & 2.70 \\
\hline $\begin{array}{l}\text { Mean operative time } \\
\text { (minutes) }\end{array}$ & 42 & 12.56 \\
\hline Bleeding (ml) & 25 & 12.90 \\
\hline Conversion to open surgery & \multicolumn{2}{|r|}{ No } \\
\hline $\begin{array}{l}\text { Deaths during } \\
\text { post-operative period }\end{array}$ & \multicolumn{2}{|r|}{ No } \\
\hline
\end{tabular}

\section{Discussion}

Laparoscopic Sleeve Gastrectomy (LSG) is currently an established method in the therapy of morbid obesity, is indicated as an alternative to Roux-en-Y Gastric Bypass (RYGB) for patients with morbid obesity with surgical indication for bariatric surgery and causing a significant excess weight loss, improvement in co-morbidities and drug reduction ${ }^{3,6,11}$. Although a well-established technique, it is still considered an advanced surgery, which requires long-surgeon training (long learning curve) to result in a standard procedure in each hospital. A higher rate of complications is statistically significant in the first cases of each surgeon ${ }^{12}$. The complications of LSG can be severe and cause significant morbidity and mortality. Postoperative bleeding and gastric stapling dehiscence can lead to reoperations and provide hospitalization for many weeks or months. Because of its simplicity IGSG is a faster procedure that might reduce operative time, possibly decreasing the complications rate and the difficulty of the procedure in the clinical set.

LSG is commonly performed through multiple ports, however, with the advent of single port surgery and natural orifice transluminal endoscopic surgery (NOTES), new procedures have been studied to result in decreased wound-related morbidity. Ramos et al performed the first series of NOTES transvaginal sleeve gastrectomy with good early postoperative results and no complications ${ }^{13}$. Using a direct access to the gastric cavity, held initially by surgeon groups in Japan, China and South Korea ${ }^{14,15}$, the intragastric technique with single port became an alternative in the treatment of selected benign and malignant gastric tumors. The technique replaces some indications for submucosal endoscopic resection (SMR) and traditional surgery. The prior exteriorization of the gastric wall to access the gastric lumen without abdominal laparoscopy was introduced by Zorron et $a l .{ }^{16}$ to perform single-site gastrostomy, and posteriorly using the approach to perform intragastric single port to remove large tumors. The procedure described here, 
Intragastric Single Port Sleeve Gastrectomy (IGSG), is an alternative technique for formal sleeve gastrectomy. In addition, it is technically feasible to perform internal plication of the greater curvature, including the fundus, thus could also be indicated for replacing a hypothetical indication for gastric greater curvature plication in obese patients, obviating the need for surgically desvascularize the stomach. Prior exteriorization of the gastric wall precludes formal abdominal laparoscopy and avoids entering the peritoneal cavity. The whole technique implies in simple percutaneous transgastric access and the procedure is entire intraluminal. The procedure is performed only inside the single port and permits also oversewing of the stapling line internally and bipolar hemostasis if needed.

IGSG is a procedure that does not require gastric devascularization of the great curvature, therefore is not necessary the assistant help to retract the stomach to start the stapling. In contrast to the conventional technique which is usually performed with two assistants, IGSG does not require more than one assistant for the realization of the procedure. The position of assistant can change according to the surgeon's needs because the incision is medial and the monitor is located behind the head of the patient. The required laparotomy to remove the specimen in LSG is usually accomplished by extending the incision through one of the trocars to 4 to $5 \mathrm{~cm}$. This incision is sometimes painful and involves potential morbidity of postoperative incisional hernias, wound infection and obstruction due to intestinal adhesions even years after the procedure ${ }^{13}$. With IGSG, direct access through a Single Port platform enables the performance of the sleeve without extensive dissection required in intra-abdominal surgical access. The specimen may be extracted transorally through the flexible endoscope or through the single port device. The exteriorization of the stomach though a small orifice in an obese patient could be clinically difficult, and sometimes, not feasible. Another limitation for clinical application in patients with known hiatal hernia represent a formal contraindication for the procedure, because of inadequate treatment of the hernia by the technique and potential severe reflux after sleeve gastrectomy. Potential disadvantages of this technique are the necessary opening of the gastric lumen involving contamination and the possibility of gastric wall haematoma by not performing previous control of the vascularization. In addition, potential injury to organs in vicinity to the gastric wall as the splenic capsule and short gastric vessels, the transverse colon and left lobe of the liver are matter of concern for future clinical applications. Finally, inadequate evaluation of the anatomy and incomplete gastric or fundic resection have also to be evaluated for the clinical effectivity of this new alternative method.

\section{- Conclusions}

Intragastric Single Port Sleeve Gastrectomy with prior exteriorization of the gastric wall is technically feasible and reproducible in an animal experimental set. It may represent an alternative method for laparoscopic sleeve gastrectomy and for endolumenal therapeutic methods for morbidly obese patients. However, the clinical feasibility and safety of this innovative therapy still may be proven in pilot studies in the morbid obese population.

\section{References}

1. Regan JP, Inabnet WB, Gagner $M$. Early experience with two-stage laparoscopic Roux-en-Y gastric bypass as an alternative in the supersuper obese patient. Obes Surg. 2003;13:861-4. doi: 10.1381/096089203322618669.

2. Gumbs AA, Gagner M, Dakin G, Pomp A. Sleeve gastrectomy for morbid obesity. Obes Surg. 2007;17:962-9. PMID: 17894158.

3. Himpens J, Dobbeleir J, Peters G. Long-term 
results of laparoscopic sleeve gastrectomy for obesity. Ann Surg. 2010;252(2):319-24. doi: 10.1097/SLA.0b013e3181e90b31.

4. Lalor PF, Tucker ON, Szomstein S, Rosenthal RJ. Complications after laparoscopic sleeve gastrectomy. Surg Obes Relat Dis. 2008;4:338. doi: 10.1016/j.soard.2007.08.015.

5. Jurowich C, Thalheimer A, Seyfried F, Fein M, Bender G, Germer CT, Wichelmann C. Gastric leakage after sleeve gastrectomy- clinical presentation and therapeutic options. Langenbecks Arch Surg. 2011;396:981-7. doi: 10.1007/s00423-011-0800-0.

6. Rosenthal RJ. International sleeve gastrectomy expert panel consensus statement: Best practice guidelines based on experience of $>12,000$ cases. Surg Obes Relat Dis. 2012;8(1):8-19. doi: 10.1016/j. soard.2011.10.019.

7. Lopez-Nava G, Galvao MP, Da BautistaCastaño I, Jimenez A, De Grado T, FernandezCorbelle JP. Endoscopic sleeve gastroplasty for the treatment of obesity. Endoscopy. 2015;47(5):449-52. doi: 10.1055/s-00341390766.

8. Saber AA, Elgamal $\mathrm{MH}$, Itawi EA, Rao AJ. Single incision laparoscopic sleeve gastrectomy (SILS): a novel technique. Obes Surg. 2008;18:1338-42. doi: 10.4103/09729941.72397.

9. Mittermair R, Pratschke J, Sucher R. Singleincision laparoscopic sleeve gastrectomy. Am Surg. 2013;79(4):393-7. PMID: 23574850.

10. Zhu Jf, Xin Y, Ma Yz, Li Xx, Hu H. Singleport laparoscopic intragastric surgery using
SILS port: a feasibility study on a porcine model. Surg Innov. 2012;19(3):236-40. doi: $10.1177 / 1553350611427924$.

11. Gagner M, Gumbs AA, Milone L, Yung E, Goldenberg L, Pomp A. Laparoscopic sleeve gastrectomy for the super-super-obese (body mass index $>60 \mathrm{~kg} / \mathrm{m}^{2}$ ). Surg Today. 2008;38(5):399-403. doi: 10.1007/s00595007-3645-y.

12. Brethauer S. Sleeve gastrectomy. Surg Clin North Am. Elsevier Inc. 2011;91(6):1265-79. doi: 10.1111/j.1469-0691.2012.03852.

13. Ramos AC, Zundel N, Neto MG, Maalouf M. Human hybrid NOTES transvaginal sleeve gastrectomy: initial experience. Surg Obes Relat Dis. 2008;4(5):660-3. doi: 10.1016/j. soard.2008.06.009.

14. De Vogelaere K, Van De Winkel N, Simoens C, Delvaux G. Intragastric SILS for GIST, a new challenge in oncologic surgery: first experiences. Anticancer Res. 2013;33(8):3359-63. doi: 0250.7005/2013.

15. Wu SD, Kong J, Su Y, Fan Y. Safety and application of transumbilical singleincision laparoscopic gastrectomy for GIST: SILS in benign gastric disease. Surg Innov. 2013;20(4):365-9. doi: $10.1177 / 1553350612455224$.

16. Zorron R, Cazarim D, Flores D, Meyer CAf, Castro Lm, Kanaan E. Single Access Gastrostomy (SAG) dispenses endoscopy or laparoscopy: a simple method under local anesthesia. Surg Innov. 2009;16(4):337-42. doi: $10.1177 / 1553350609351694$.

\section{Correspondence:}

André Lacerda de Abreu Oliveira

Laboratório de Sanidade Animal

Centro de Ciências e Tecnologias Agropecuárias,

UENF Darcy Ribeiro

Avenida Alberto Lamego, 2000

28013-602 Campos dos Goytacazes - RJ Brasil

Tel.: (55 22)2748-6084 / (21)99397-0795

andrevet@uol.com.br

Received: Sep 16, 2017

Review: Nov 17, 2017

Accepted: Dec 20, 2017
Conflict of interest: none

Financial source: none

\begin{abstract}
${ }^{1}$ Research performed at Laboratory of Animal Health and Laboratory of Cell and Tissue Biology, Animal Experimentation Division, Universidade Estadual do Norte Fluminense Darcy Ribeiro (UENF), Campos dos GoytacazesRio de Janeiro, Brazil.
\end{abstract}

